Musées, Patrimoine et Culture scientifiques et techniques

$153 \mid 2014$

mai-juin 2014

\title{
Récolement et diffusion dans les bases de données spécialisées
}

Marie-Dominique Wandhammer et Nicolas Morel

\section{OpenEdition \\ Journals}

Édition électronique

URL : http://journals.openedition.org/ocim/1402

DOI : $10.4000 /$ ocim. 1402

ISSN : 2108-646X

Éditeur

OCIM

Édition imprimée

Date de publication : 25 juin 2014

ISSN : 0994-1908

Référence électronique

Marie-Dominique Wandhammer et Nicolas Morel, «Récolement et diffusion dans les bases de données spécialisées », La Lettre de l'OCIM [En ligne], 153 | 2014, mis en ligne le 25 juin 2016, consulté le 01 mai 2019. URL : http://journals.openedition.org/ocim/1402 ; DOI : 10.4000/ocim.1402 


\title{
Récolement et diffusion dans les bases de données spécialisées
}

\author{
Marie-Dominique Wandhammer et Nicolas Morel *
}

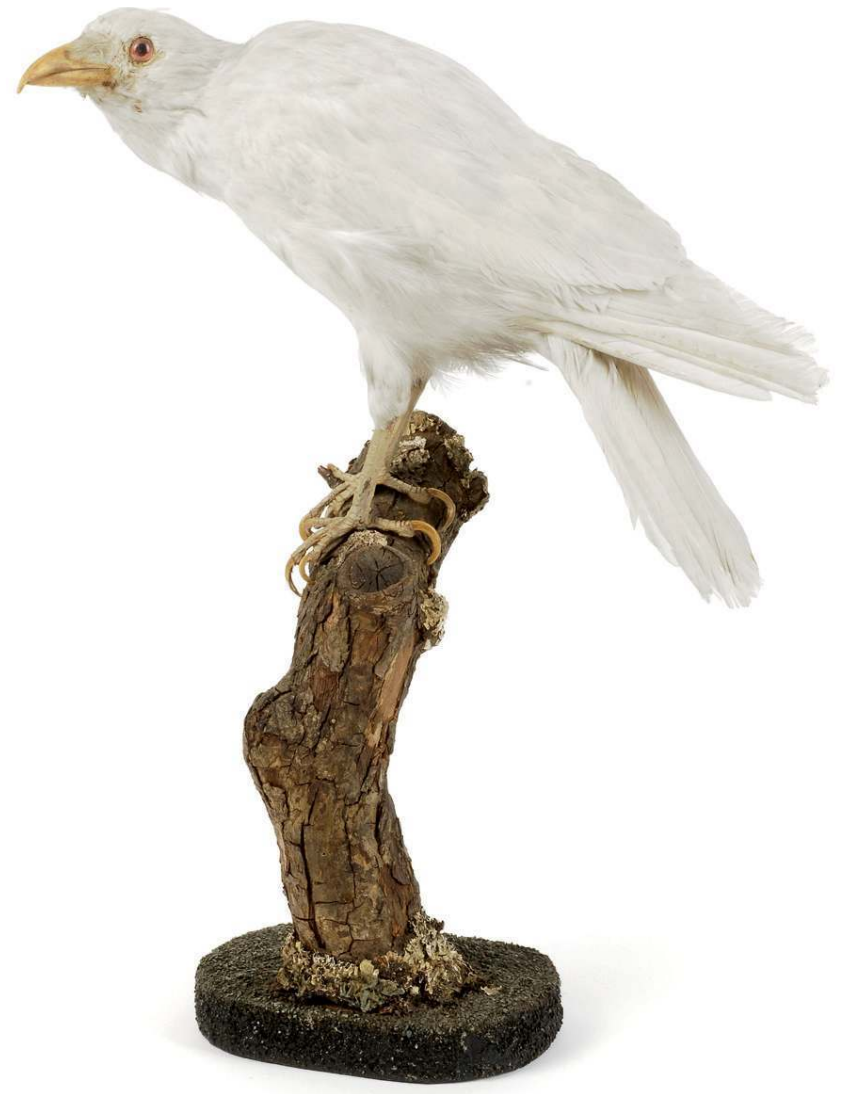

Corneille noire albinos, Corvus corone (inv. Ave06525), spécimen de la collection d'ornithologie du musée zoologique de Strasbourg, versée dans GBIF. (-) Musées de Strasbourg/Mathieu Bertola

* Marie-Dominique Wandhammer est conservateur du musée zoologique de Strasbourg marie-dominique.wandhammer@strasbourg.eu

Nicolas Morel est directeur du musée Vert, muséum d'Histoire naturelle du Mans nicolas.morel@ville-lemans.fr
L'inventaire et le récolement permettent

au personnel de conservation des musées

$d^{\prime}$ acquérir une connaissance approfondie des

collections et de compléter les informations

qui y sont liées. Quelles suites donner à cette

mission qui représente un investissement

considérable pour les institutions muséales?

Une fois les collections récolées, les demandes de consultations, de prêts ou d'envois de données à des fins scientifiques constituent de bons indicateurs de leur intérêt, mais encore faut-il que les inventaires réalisés aient une bonne visibilité. La conservation en interne de centaines de milliers de données numériques semble en effet inadaptée aux enjeux et aux possibilités actuelles.

L'une des voies ouvertes par les TIC concerne la mise en ligne de bases de données permettant l'accès à des millions d'informations pour le plus grand nombre. Les collections d'Histoire naturelle, qui suscitent un regard sur l'évolution du vivant et constituent un des outils indispensables à la recherche menée dans le domaine de la biodiversité, sont notamment concernées. Cette mise en ligne correspond à une valorisation aujourd'hui indispensable des collections patrimoniales afin de leur permettre d'exister au-delà des limites des structures de conservation.

La publication sur des bases de données françaises ou internationales ouvre d'importantes possibilités : bases généralistes, bases thématiques, métadonnées, bases axées sur la taxonomie, sur les collections, les observations... Difficile parfois de s'y retrouver et de faire des choix au sein d'un paysage complexe. Il faut néanmoins savoir que bon nombre de bases ont des liens entre elles et que, sans forcément en avoir connaissance, les données communiquées sont automatiquement intégrées au sein d'autres programmes. 


\section{Les bases de données classées par typologie}

\section{Zoologie, Botanique}

\section{GBIF}

La base mondiale Global Biodiversity Information Facility a pour vocation de mettre à disposition toute l'information connue sur la biodiversité. Elle regroupe des données primaires (plus de 1400000 espèces recensées) sur la biodiversité (des gènes aux écosystèmes) provenant de 580 contributeurs et en particulier des musées. Les données se répartissent entre les informations provenant des collections et les observations sur le terrain. Le GBIF propose également des métadonnées en présentant les caractéristiques de la collection ou du dispositif d'observation. En France, les muséums d'Autun, d'Aix-en-Provence, de Clermont-Ferrand, du Mans, de Bourges, de Nice et de Strasbourg ont versé des données partielles de leurs inventaires. Le GBIF France est géré par le Muséum national d'Histoire naturelle (MNHN).

www.gbif.fr

Coordinateurs GBIF France : Éric Chenin (eric.chenin @ird.fr) Régine Vignes (regine.vignes_lebbe@upmc.fr) Communication et gestion des ressources : Anne-Catherine Archambeau (archambeau@gbif.fr)

\section{MaNIS, ORNIS, HerpNet, FishNet}

Ces quatre bases spécialisées, créées sous l'impulsion de muséums d'Histoire naturelle et d'universités, regroupent respectivement des données de collections de mammalogie, d'ornithologie, d'herpétologie et d'ichtyologie. Elles ont essentiellement pour but de faciliter l'accessibilité aux données des spécimens et d'en favoriser la valorisation. Ces données ont été rassemblées sur VertNet, un portail global consacré aux vertébrés. Elles sont automatiquement reversées sur le portail GBIF puisque, depuis septembre 2013, VertNet est devenu participant associé au réseau GBIF. Aucun musée français ne participe à ces quatre bases. http://manisnet.org

http://ornis2.ornisnet.org - Référent: Town Peterson (town@ku.edu)

www.herpnet.org - Référent : Carol Spencer (atrox@berkley.edu)

www.fishnet2.net - Référent : Nelson E. Rios (nrios@tulane.edu)

http://portal.vertnet.org - Référent : Lara Russel (larussel@ku.edu)

\section{BioCASE}

Ce réseau européen de collections biologiques (Biological Collection Access Service for Europe) permet un accès unifié aux bases de données stockant des informations liées aux collections et aux observations de terrain (d'origine européenne). Après la création d'un accès aux métadonnées (actuellement BCI, Biodiversity
Collections Index), un accès aux données unitaires a vu le jour. À noter : BioCASE est un fournisseur de données pour le GBIF. Un fournisseur de données sur BioCASE est ainsi automatiquement fournisseur pour le GBIF. Au niveau français, y sont référencées des données des muséums d'Autun, d'Aix-en-Provence, de Bourges, de Clermont-Ferrand, du Mans, de Nice et de Strasbourg.

http://biocase.org

Référent : Jörg Holetschek (j.holetschek@bgbm.org)

\section{INPN}

L'Inventaire National du Patrimoine Naturel est une banque nationale de données de référence sur la biodiversité et la géodiversité française. Son but est de centraliser, valider et diffuser les informations relatives au patrimoine naturel (terrestre et marin) de la France. Elle prend en compte les espèces végétales et animales, les milieux naturels et le patrimoine géologique. Cet outil permet l'expertise et l'élaboration de stratégies de conservation.

L'INPN recense des données actuelles, auxquelles s'ajoutent les données anciennes issues des collections qui permettent leurs mises en perspective. Complémentaires des observations fournies par les réseaux naturalistes, elles donnent ainsi un autre regard sur l'évolution des écosystèmes.

L'INPN est la plate-forme nationale du SINP (Système d'Information sur la Nature et les Paysages) qui constitue un dispositif partenarial entre le ministère de l'Écologie, du Développement durable, des Transports et du Logement, les associations, les collectivités, les services de l'État comme l'ONF (Office National des Forêts), l'ONCFS (Office National de la Chasse et de la Faune Sauvage), l'ONEMA (Office National de l'Eau et des Milieux Aquatiques), IFREMER (Institut Français de Recherche pour l'Exploitation de la Mer)... Il s'agit d'un travail collaboratif pour produire, gérer, traiter, valoriser et diffuser des données relatives à la biodiversité et la diversité des paysages. Dans ce cadre, afin de permettre une communication entre tous ces acteurs, le MNHN a développé un référentiel taxonomique, TAXREF, qui fournit des normes communes de référence et permet ainsi l'interopérabilité des bases. http://inpn.mnhn.fr

Référent : Laurent Poncet (poncet@mnhn.fr)

\section{EBEAC}

eBEAC rassemble une communauté de conservateurs de collections ornithologiques. Cette communauté est à l'origine d'une base de données de collections (métadonnées), hébergée par le Natural History Museum de Londres. Cette base décrit sommairement le contenu des fonds ornithologiques de 262 musées et institutions européennes dont, pour la France, les muséums d'Autun, Bordeaux, Dijon, Grenoble, La Rochelle, Le Havre, Le Mans, Lille, Lyon, Marseille, Montauban, 
Nancy, Nantes, Nice, Perpignan, Rouen, Strasbourg, Toulouse, ainsi que le MNHN.

www.nhm.ac.uk/research-curation/scientific-resources/collections/zoological-collections/ebeac/index.html

Référent : Douglas Russell (d.russell@nhm.ac.uk)

\section{TELA BOTANICA}

Dans le cadre du programme eReColNat, l'association Tela Botanica et le PRES Clermont-Université ont pour mission de recenser les herbiers publics et privés de France. Du point de vue des collections publiques conservées dans des muséums, la base "Herbiers " (CoEL) rassemble des métadonnées provenant des muséums d'Aix-en-Provence, Angers, Autun, Bourges, Clermont-Ferrand, Colmar, Gap, Grenoble, Le Mans, Lyon, Montbéliard, Nantes, Nice, Toulon-Var, Toulouse et du MNHN. Au total, 37 musées français ont versé des collections botaniques, soit 479 herbiers actuellement décrits.

www.tela-botanica.org/page:herbiers_recherche

Référent: Louise Boulangeat (louise@tela-botanica.org)

\section{SONNERAT-BRYOMICO}

La base Sonnerat-Bryomico du Réseau des Herbiers de France est consacrée à la botanique et à la mycologie. Gérée et organisée autour des collections du Muséum national, cette base est notamment orientée vers les collections botaniques historiques. Des conventions sont signées entre le MNHN et les institutions participantes, notamment les muséums d'Aixen-Provence et de Dijon. Accessible en ligne, cette base de données compte aujourd'hui plus d'un million de parts d'herbiers inventoriées.

http://science.mnhn.fr/institution/mnhn/search www.mnhn.fr/fr/collections/bases-donnees-scientifiques/reseau-herbiers-france

Référent : Marc Pignal (pignal@mnhn.fr)

Il existe également dans le domaine de la botanique, les bases de données des différents Conservatoires Botaniques Nationaux (CBA) comme la base eCalluna du CBN de Brest.

\section{Paléontologie - Géologie}

\section{TRANS'TYFIPAL ${ }^{\circledR}$}

Le programme national Trans'Tyfipal ${ }^{\circledR}$ est spécialisé dans l'inventaire des «types » et figurés paléontologiques conservés en France (voir le texte de Jérôme Thomas, p. 84).

\section{GEOCASE}

Geosciences Collection Access Service est un programme européen dont l'objectif est d'agréger des bases de données de collections consacrées à la paléontologie et à la géologie. Il est l'équivalent de BioCASE pour les sciences de la Terre. Il permet d'interroger pour le moment les collections de quatorze institutions partenaires (Allemagne, pays d'Europe Centrale et des Pays Baltes) pour un total supérieur à un million de données. Aucun muséum français ne participe actuellement à GeoCASE.

www.geocase.eu/

Référent : Wolfgang Kiessling

(wolfgang.kiessling@fau.de)

\section{Sciences et techniques}

\section{PATSTEC}

Le site du programme national PATSTEC (Patrimoine Scientifique et Technique Contemporain, www.patstec.fr) constitue un catalogue multimédia présentant à la fois un inventaire documenté d'objets et des médias (photographies, interviews de chercheurs, savoirfaire vidéos, animations) du patrimoine scientifique et technique contemporain.

Lopérateur de ce programme est le musée des Arts et Métiers. Les établissements ressources et porteurs de missions locales fonctionnant en réseau sont les universités et les organismes de recherche, les industries, les centres régionaux des Cnam, ainsi que les musées. Sont associés des partenaires nationaux comme le CEA, le CERN, les musées d'EDF, de Météo France et le CNRS.

Le site web national PATSTEC donne actuellement accès à 5537 objets et aux documents média qui leurs sont associés. Ces instruments se repartissent temporellement entre 1750 et la période actuelle, néanmoins leur grande majorité est postérieure à 1875. La base de données outil professionnel du réseau, comprend plus de 15000 fiches et 35000 médias.

Les muséums de Clermont-Ferrand et de Lyon, ainsi que le musée du Temps de Besançon ont fourni à ce jour 3800 données à cette base.

www.patstec.fr/PSETT

Référents: Catherine Cuenca

(catherine.cuenca@univ-nantes.fr)

Valérie Joyaux (valerie.joyaux@univ-nantes.fr)

\section{Ethnologie - Préhistoire}

\section{JOCONDE}

Le catalogue Joconde, géré par le service des musées de France, regroupe près de 500000 notices d'objets de toute nature (archéologie, beaux-arts, ethnologie, histoire...). Cette base a pour objectif de recenser le patrimoine culturel mobilier des « Musées de France». Actuellement, environ 320 musées ont versé une partie de leurs collections dans cette base par le biais de conventions de partenariat signées avec le ministère de la Culture et de la Communication.

Du point de vue des muséums, des collections ethnologiques ont été intégrées par Besançon, Cherbourg, 
Lille et Rouen pour un total de 716 notices (sur un total de 44960 objets ethnologiques présents dans Joconde), ainsi que 199 dessins du fonds Lesueur par le muséum du Havre.

www.culture.gouv.fr/documentation/joconde/fr/

Référent : Laurent Manœuvre

(laurent.manoeuvre@culture.gouv.fr)

\section{Les bases de données et les collections strasbourgeoises}

Les premières collections à rejoindre une banque de données ont été les spécimens-« types » d'ichtyologie. L'inventaire de cette collection a été finalisé quand le MNHN, par la voix de Jean-Claude Hureau, a fait la demande d'intégrer les spécimens-«types » dans la base GICIM ou Gestion Informatisée des Collections Ichtyologiques des muséums d'Histoire naturelle de France. Jean-Claude Hureau procéda ainsi avant l'heure à un récolement et à une vérification sur place des spécimens. Créée en 1982, GICIM rassemble les données sur les spécimens-«types » des muséums de Lyon, La Rochelle, Monaco et Strasbourg et depuis la fin des

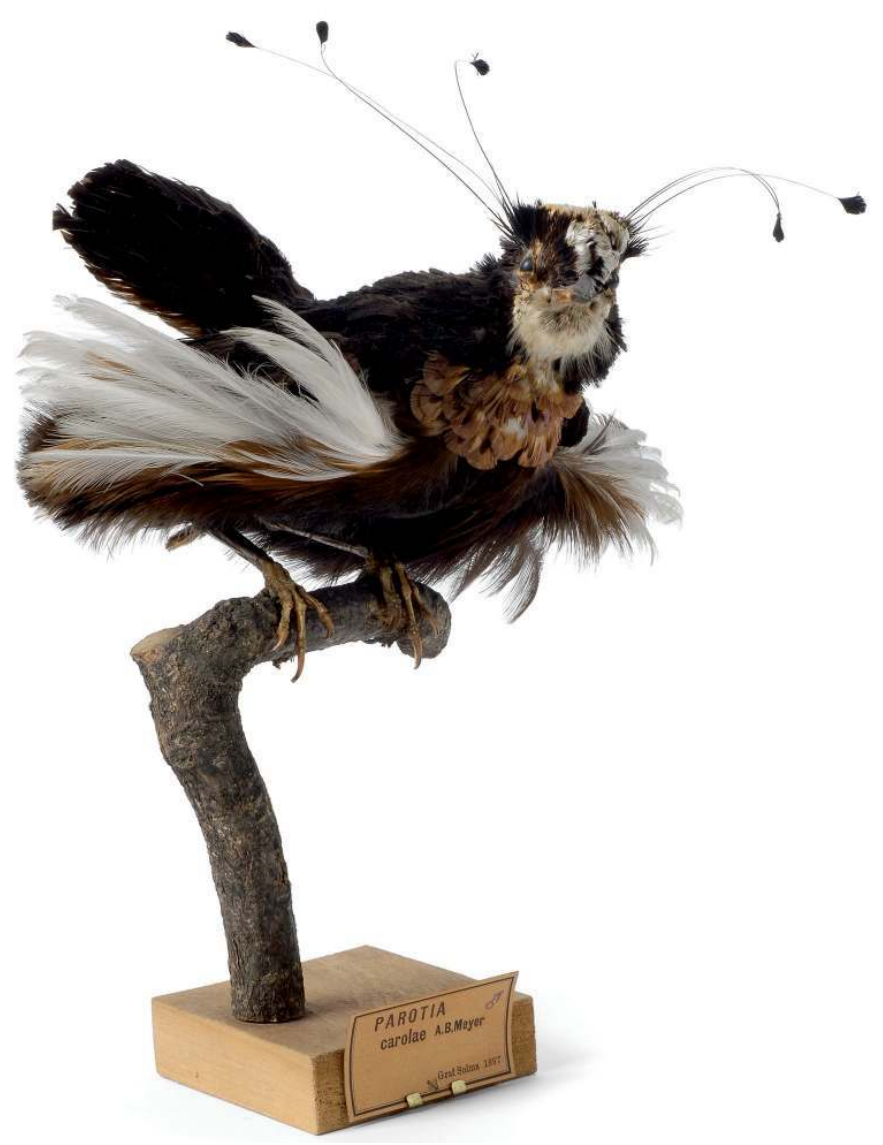

Paradisier de Carola, Parotia carolae (inv.Ave04522), spécimen de la collection d'ornithologie du musée zoologique de Strasbourg, versée dans GBIF. (c) Musées de Strasbourg/Mathieu Bertola années 1990 la collection du MNHN. Les données sont référencées dans les bases de taxonomie telles Fishbase et Fishnet2 et apparaissent sous l'acronyme du musée (MZS par exemple) avec comme référence GBIFMNHN

Dans d'autres bases de taxonomie, on retrouve des données concernant les spécimens-« types » comme par exemple dans WoRMS (World Register of Marine Species) où sont référencés quelques vint-cinq crustacés et éponges, reprises quant à elles dans World Porifera Database.

Le récolement faisant son chemin, trois autres inventaires ont été versés dans le GBIF : celui de la collection d'ornithologie et ceux des cnidaires et des spongiaires. L'inventaire des cnidaires avait bénéficié de la révision d'un spécialiste de ce groupe. Les données étant majoritairement en langue allemande, beaucoup d'erreurs s'y étaient glissées, que le récolement a permis de rectifier. Comme le suggère justement un de nos collègues, nous aurions dû transmettre des données retravaillées et non brutes comme nous l'avons fait, certaines d'entre elles étant difficilement exploitables en l'état. À terme, ces données devraient être complétées par des photographies qui alimenteront la bibliothèque numérique Europeana.

La base GICIM a eu des retours immédiats de consultation des spécimens-« types », tout comme les quelques spécimens référencés dans WoRMS. Pour les autres versements de données, il est difficile d'apprécier leurs effets. Les chercheurs ne mentionnent en effet que rarement dans leurs demandes de prêts ou d'informations, comment ils ont eu connaissance de nos collections. Parfois, il est fait référence à GBIF, mais la plupart du temps ce sont des publications, même anciennes, qui les amènent à nous questionner sur la présence de telle ou telle espèce dans nos collections. Difficile aussi d'avoir une idée du taux de consultation des données dans les différentes bases et de leur utilisation. Notre politique à l'heure actuelle est de continuer à alimenter les bases, mais aussi de publier sur le site des musées de la Ville de Strasbourg, une fois nos inventaires revus.

\section{Les bases de données et les collections mancelles}

Le musée Vert, muséum d'Histoire naturelle du Mans, n’a commencé à fournir des données à des bases nationales qu'à partir de 2010. Face à la quasi-absence de recensements anciens des collections, les opérations d'inventaire et de récolement ont généralement été liées. Les données ornithologiques ont été extraites de la base du musée (logiciel SN-Base), converties en fichier Excel puis transmises au GBIF en 2012. Cette opération fastidieuse devrait être reproduite plus facilement 


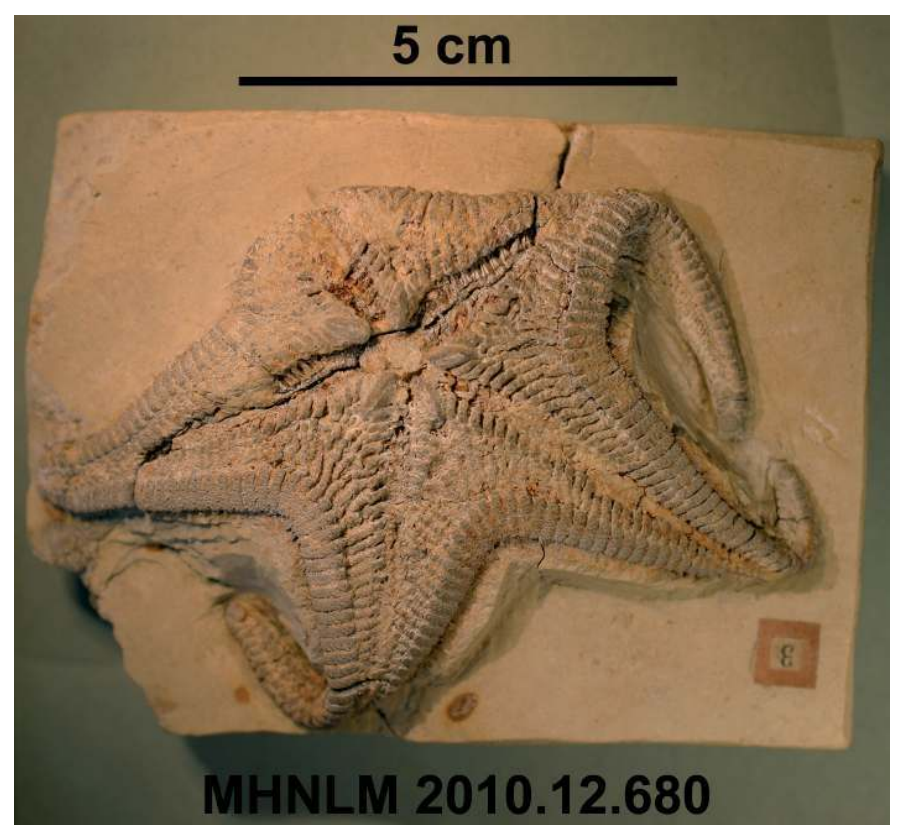

Holotype de l'étoile de mer Tethyaster guerangeri, Breton, 1995, du Cénomanien du Mans : un des types et figurés des collections du musée Vert, versé dans Trans'Tyfipal ${ }^{\circledR}$. ○ Musée Vert du Mans

à l'avenir pour les autres collections du musée. Il faut noter certaines discordances entre les données de collection et les demandes du GBIF. C'est notamment le cas concernant les provenances géographiques, généralement traitées en lieu-dit/commune dans SN-Base, mais devant être reversées en latitude/longitude afin d'être consultables au sein du GBIF.

Les données des 900 « types » et figurés des collections paléontologiques ont été fournies à Trans'Tyfipal ${ }^{\circledR}$. Leur équipe de prise de vue a effectué un inventaire photographique complet de cet ensemble en novembre 2013.
Pour mémoire, de nombreuses autres bases de données existent mais n'ont pas de rapport direct avec l'inventaire et le récolement des collections PSTN (Patrimoine Scientifique, Technique et Naturel) des musées. Il s'agit notamment d'ISIS (animaux de zoos), PLANT SEARCH (jardins botaniques), DIGICOL (archives et bibliothèques), EuroBioBank (ADN, cellules et tissus), BIOGeomancer (géoréférencement des données), DNA Bank Network (banques d'ADN), Biofresh (conservation des écosystèmes d'eau douce), LIFEWATCH (orienté recherche sur le thème de la biodiversité), NUMES (corpus numérisés dans l'enseignement supérieur et la recherche), PALISSY (patrimoine mobilier des monuments historiques), SciColl (programme international de promotion des collections scientifiques), SYNTHESIS (accessibilité, mise en réseau et activités de recherche), ainsi que des bases taxonomiques comme algaebase, The Plant List, FishBase, Species 2000 ou PaleoBiologyDataBase.

Les auteurs tiennent à remercier pour leur aide: AnneCatherine Archambeau (GBIF France), Ludovic Besson (muséum de Bourges), Louise Boulangeat (Tela Botanica), Joël Clary (musée des Confluences, Lyon), Catherine Cuenca (PATSTEC), Jörg Holetschek (BioCASE), Laurent Mancuvre (Joconde), Wolfgang Kiessling (GeoCASE), Sophie Pamerlon (GBIF France), Margaux Pizzo (SYNTHESIS), Laurent Poncet (INPN) et Thomas Rouillard (muséum d'Angers).

\section{Bibliographie}

Charon, P. Les inventaires du Patrimoine, Scientifique, Technique et Naturel, La Lettre de l'OCIM, n¹34, mars-avril 2011, pp. 46-51. 